\title{
First clinical application of radiofrequency identification (RFID) marking system - Precise localization of a small lung nodule
}

Toshihiko Sato, MD, PhD, ${ }^{\mathrm{a}}$ Yojiro Yutaka, $\mathrm{MD}, \mathrm{PhD},{ }^{\mathrm{b}}$ Tatsuo Nakamura, $\mathrm{MD}, \mathrm{PhD},{ }^{\mathrm{c}}$ and Hiroshi Date, MD, $\mathrm{PhD}^{\mathrm{b}}$

\section{ABSTRACT}

Objectives: Precise small lung nodule resection is challenging in minimally invasive thoracoscopic surgery. Various methods that help surgeons to locate the target nodule have been devised; however, the ideal way that satisfies the demand has not yet been realized. We have developed and applied a novel marking system to localize small lung nodules for the first time in humans.

Methods: A radiofrequency identification tag $(1.8 \mathrm{~mm}$ in diameter and $7 \mathrm{~mm}$ in length) that can communicate with a wand-shaped antenna (10 $\mathrm{mm}$ in diameter) from the distance of $3 \mathrm{~cm}$ was prepared. The tag was delivered adjacent to a 7-mm subsolid nodule in the right lower lobe of a patient under cone beam computed tomography guidance and video-assisted thoracoscopic surgery wedge resection was subsequently performed.

Results: The delivery of the tag was smooth, and the tag was almost immediately detected by the antenna. Wedge resection was successfully performed with the guiding signal from the tag.

Conclusions: We have so far demonstrated that this technology could be applicable for small lung nodule detection in preclinical studies. In this first clinical experience, this system proved to provide accurate positional information of small lung nodules with depth. (JTCVS Techniques 2020;4:301-4)

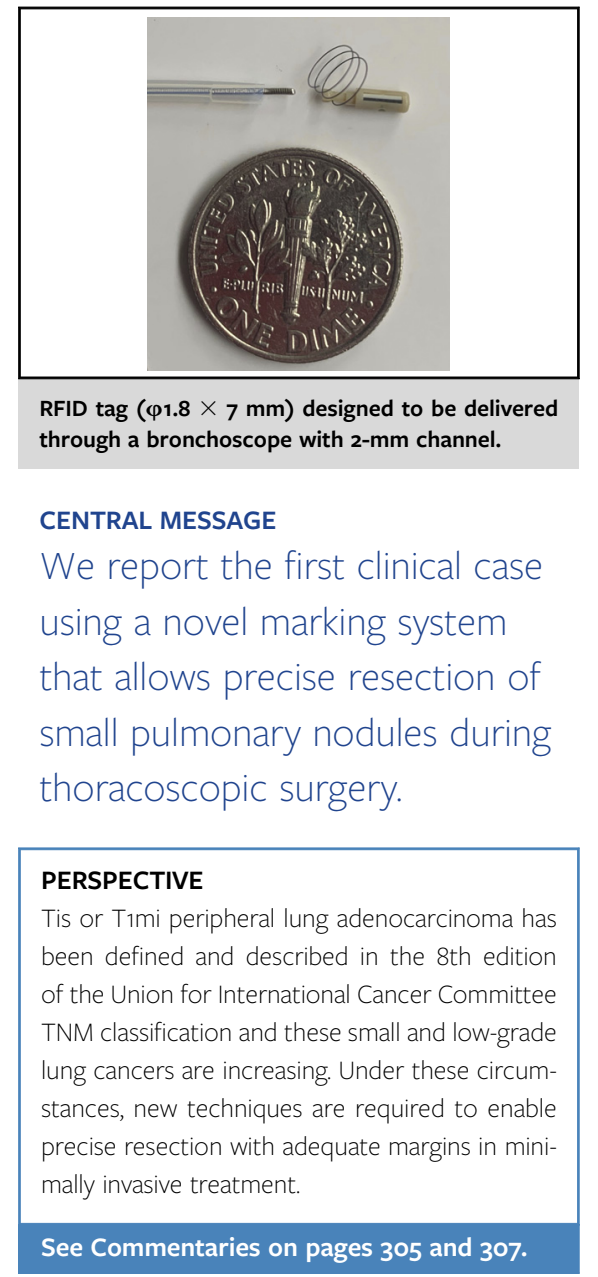

From the a Department of Thoracic, Breast and Pediatric Surgery, Fukuoka University, Fukuoka; and ${ }^{\mathrm{b}}$ Department of Thoracic Surgery and ${ }^{\mathrm{c}}$ Institute for Frontier Medical Sciences, Kyoto University, Kyoto, Japan.

Accepted for the 100th Annual Meeting of The American Association for Thoracic Surgery.

Received for publication Sept 8, 2020; accepted for publication Sept 17, 2020; available ahead of print Sept 24, 2020.

Address for reprints: Toshihiko Sato, MD, PhD, 7-45-1 Nanakuma, Jonan, Fukuoka 814-0180, Japan (E-mail: toshisato@ fukuoka-u.ac.jp).

2666-2507

Copyright (C) 2020 The Authors. Published by Elsevier Inc. on behalf of The American Association for Thoracic Surgery. This is an open access article under the CC BY-NCND license (http://creativecommons.org/licenses/by-nc-nd/4.0/).

https://doi.org/10.1016/j.xjtc.2020.09.018 


\section{Abbreviations and Acronyms}

$\mathrm{CT}=$ computed tomography

RFID $=$ radiofrequency identification

the detector and the tag by an audiovisual interface from the signal processing unit (Figure 1), and system monitor informs the surgeon by flicking the targeted tag that was previously registered in the system. The system was approved as class 3 medical device by Japanese Pharmaceuticals and Medical Devices Agency in July 2018.

\section{CLINICAL SUMMARY}

A 75-year-old female patient with a 7-mm subsolid nodule in the basal segment of the right lower lobe was referred to our department (Figure 2). Periodical thin-slice computed tomography (CT) inspection confirmed the nodule had been gradually enlarging; thus, wedge resection was planned for her. She once had developed severe pneumonia in the past, and the flattened diaphragm shadow in her chest radiograph suggested some adhesion in the thoracic cavity. To localize the nodule in the thoracoscopic procedure, a novel radiofrequency identification (RFID) marking system was employed. In the hybrid operating theater, the patient was intubated with a single-lumen endotracheal tube under general anesthesia, and an RFID tag (1.8 $\mathrm{mm}$ in diameter and $7 \mathrm{~mm}$ in length) was delivered via a bronchoscope to the adjacent of the target nodule under fluoroscopic and virtual bronchoscopic guidance. Cone-beam CT confirmed that the tag was placed $10 \mathrm{~mm}$ caudal, $5 \mathrm{~mm}$ lateral to the target. After the endotracheal tube was changed to a double-lumen tube, the patient was placed in the left decubitus position, and three $10-\mathrm{mm}$ ports were set in the 5th and 8th intercostal space. Whole lung adhesion to the thoracic wall was found. After we detached the adhesion, the wand-shaped locating detector $(10 \mathrm{~mm}$ in diameter) was introduced into the thoracic cavity to receive the signal from the RFID tag. Detection of the signal was almost immediately achieved; then, successful wedge resection was performed with secure margin of $15 \mathrm{~mm}$ (Video 1). Afterward, pathologic examination confirmed that the target nodule was papillary adenocarcinoma $(7 \mathrm{~mm})$. A signed informed consent in publishing the result and the clinical course was obtained from the patient.

\section{DISCUSSION}

Current guidelines for the incidentally detected pulmonary nodule recommend resection for suspicious pulmonary nodules $\geq 6 \mathrm{~mm}$ after a certain observation period. ${ }^{1}$ However, precise localization and resection of such small and subsolid or pure ground-glass lesion are extremely difficult without any means of preoperative marking. So far, various procedures have been attempted and applied, including placement of a hook wire and delivery of fiducials, coils, dyes, indocyanine green, contrast medium, barium, intraoperative ultrasound, and injection of radioactive tracers and their combinations through percutaneous and

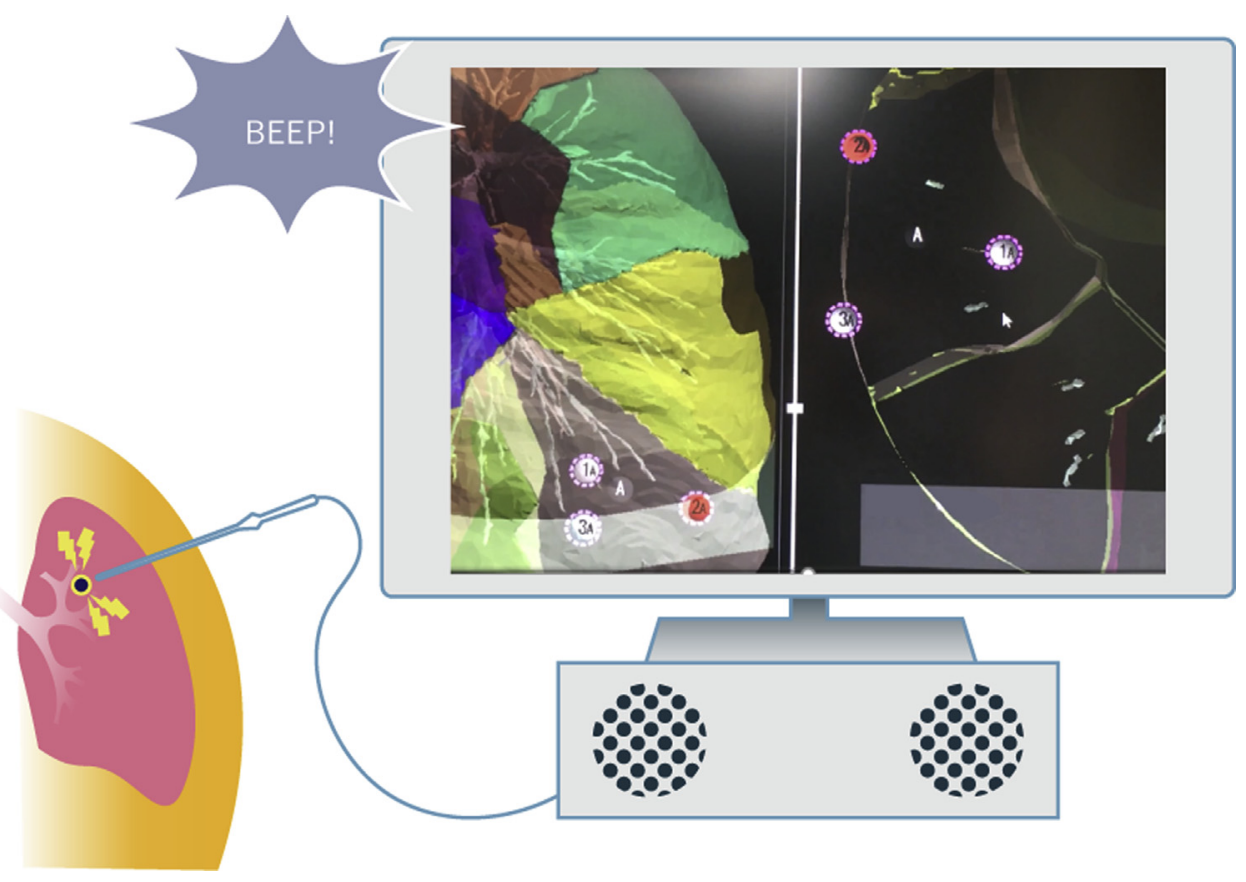

FIGURE 1. Scheme of RFID marking system. The RFID tag $(\varphi 1.8 \times 7 \mathrm{~mm})$ is designed to be delivered through a bronchoscope before resection. The surgeon determines the distance between the tag and tip by an audiovisual interface. 

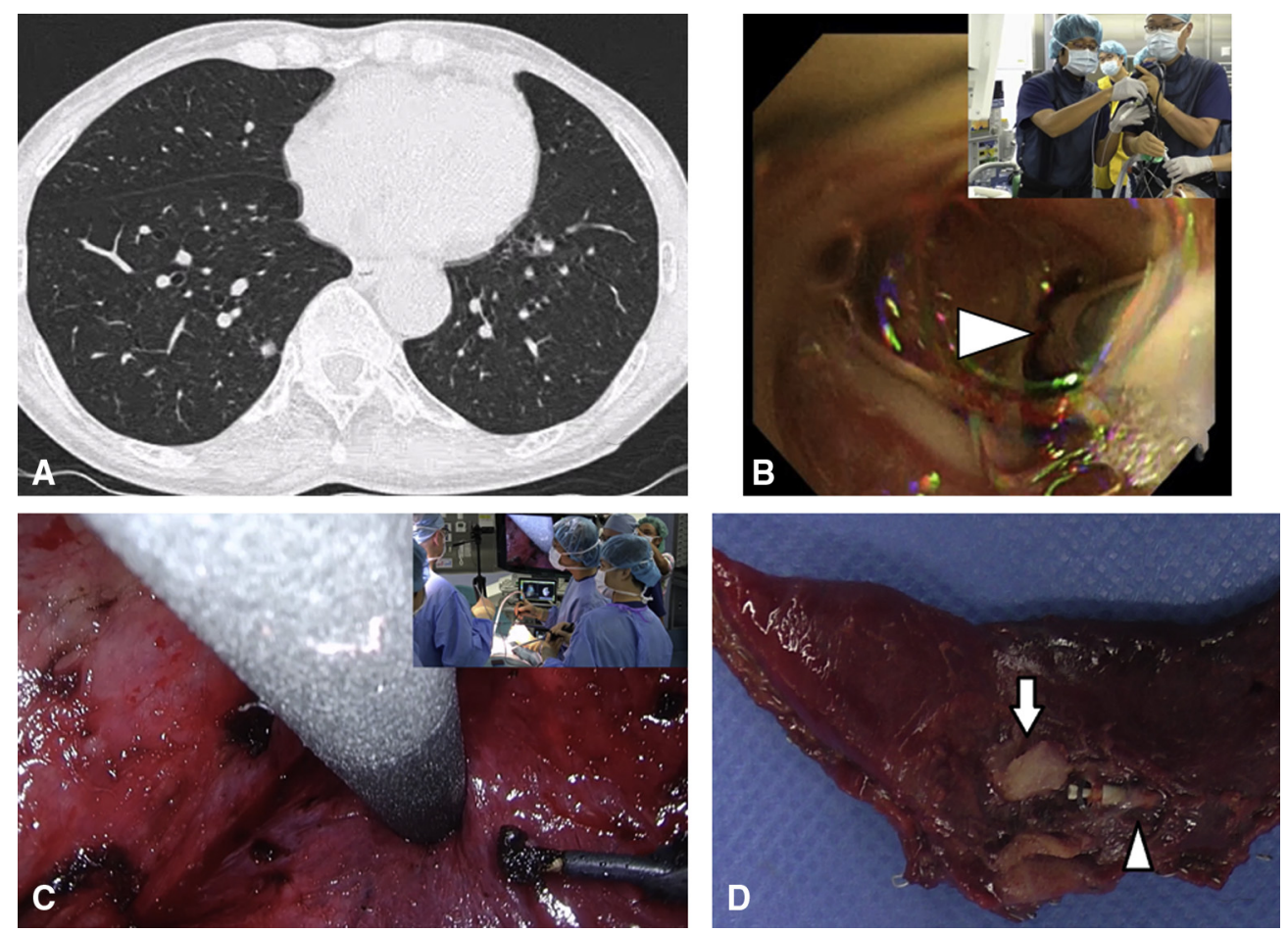

FIGURE 2. A, Preoperative CT of the case. A 7-mm subsolid nodule in the right lower lobe was detected. B, Tag delivery under fluoroscopic and conebeam CT guidance. Arrowhead, the RFID tag at the tip of the introducer. C, Receiving signal from the tag during a thoracoscopic procedure. The system informs the surgeon of the distance to the tag by audio interface (closer the tag, the higher pitch returns). The responding tag is shown by flicking in the system monitor, placed beneath the main monitor. D, Resected specimen. Arrowhead, Target nodule confirmed to be adenocarcinoma. Arrow, implanted tag.

bronchoscopic approaches. ${ }^{2}$ The multitude of reports indicate that this is one of the issues to be addressed by the modern thoracic surgeon. Lesions just beneath the pleura, even pure-ground-glass ones, might be located and removed without preoperative marking, but still some measures would be useful in determining the precise resection area (or volume). Deeper lesions $(2 \mathrm{~cm})$ are always challenging for the thoracic surgeon, and some measures should be taken to localize the target; however, they are not always

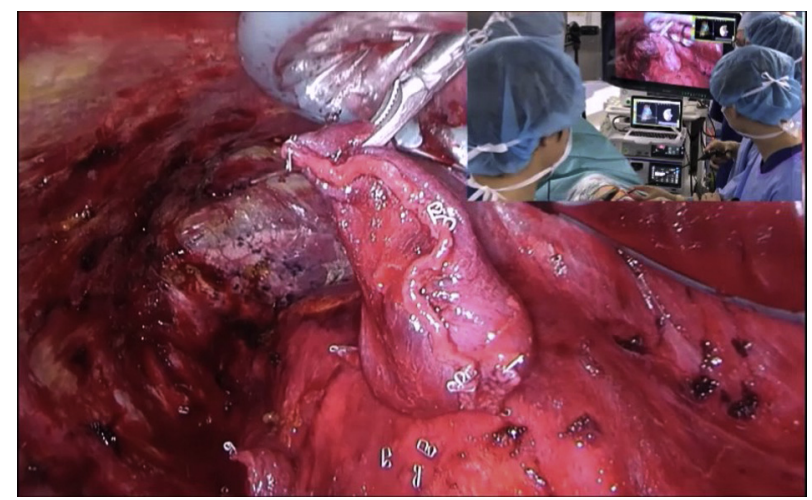

VIDEO 1. This video describes an overview of the RFID marking system and reported that a small lung nodule of $7 \mathrm{~mm}$ was accurately excised using this system. RFID, Radiofrequency identification. Video available at: https://www.jtcvs.org/article/S2666-2507(20)30528-9/fulltext. practical and may cause severe adverse event including air embolism. Among many marking measures, dye marking through a bronchoscope is well investigated and documented by Sato and colleagues. They conducted a multi-institutional prospective study investigating the efficacy of the dye marking method, which they reported in the Journal. ${ }^{3}$ They reported that insufficient dye marking was noted in $14 \%$ of patients, and the most common reason was central location, failure to show the deep margin, followed by anthracotic lung and emphysema. So far, we have demonstrated that our methodology using near-field communication could be applicable in the detection of small lung nodules and showed the deep margin precisely in preclinical studies. ${ }^{4,5}$ In these previous studies using a canine model, the tag delivered to the designated place was retained for months without dislocation, the use of a stapler caused no problems at the time of excision, and no remarkable adverse events, including pneumothorax, hemoptysis, and pneumonia, were observed.

In this first clinical experience, we were able to accurately localize the small lung nodule with this novel system despite the whole lung adhesion, which might have hindered visual localization with dye from the lung surface, and successfully performed wedge resection. This RFID lung marking system provides accurate positional information with depth because the tag was designed to be delivered 
and stay both at the subsegmental bronchus and into the lung parenchyma namely just beneath the pleura. Furthermore, since each tag has its own ID, real navigational surgery by placing multiple tags for sublobar resection is expected to be performed. ${ }^{5}$ Regarding tag placement before resection, we deliver the tag under cone-beam $\mathrm{CT}$ guidance, but a more accurate and simple delivery will be realized through multiple platforms, such as electromagnetic navigational bronchoscope and a robotic system, in the near future. We are applying this technique not only for wedge resection but also subsegmentectomy or segmentectomy and are planning to conduct a multicenter trial to prove efficacy and benefit for the patients with small lung nodules.

\section{Conflict of Interest Statement}

Drs Sato, Yutaka, and Date are receiving research support from HOGY Medical Inc, Tokyo, Japan. Dr Yutaka has an issued patent for RFID reader of positional localization. Dr Nakamura has reported no conflicts of interest.

The Journal policy requires editors and reviewers to disclose conflicts of interest and to decline handling or reviewing manuscripts for which they may have a conflict of interest. The editors and reviewers of this article have no conflicts of interest.

\section{References}

1. MacMahon H, Naidich DP, Goo JM, Lee KS, Leung ANC, Mayo JR, et al. Guidelines for management of incidental pulmonary nodules detected on CT images: from the Fleischner Society 2017. Radiology. 2017;284: 228-43.

2. Velasquez R, Martin A, Abu Hishmeh M, DeLorenzo L, Dhillon SS, Harris K. Placement of markers to assist minimally invasive resection of peripheral lung lesions. Ann Transl Med. 2019;7:360.

3. Sato M, Kobayashi M, Kojima F, Tanaka F, Yanagiya M, Kosaka S, et al. Effect of virtual-assisted lung mapping in acquisition of surgical margins in sublobar lung resection. J Thorac Cardiovasc Surg. 2018;156:1691-701.e5.

4. Kojima F, Sato T, Takahata H, Okada M, Sugiura T, Oshiro O, et al. A novel surgical marking system for small peripheral lung nodules based on radio frequency identification technology: feasibility study in a canine model. J Thorac Cardiovasc Surg. 2014;147:1384-9.

5. Yutaka Y, Sato T, Matsushita K, Aiba H, Muranishi Y, Sakaguchi Y, et al. Threedimensional navigation for thoracoscopic sublobar resection using a novel wireless marking system. Semin Thorac Cardiovasc Surg. 2018;30:230-7.

Key Words: lung cancer, small nodule, minimally invasive surgery 